\title{
The bionomics of the malaria vector Anopheles farauti in Northern Guadalcanal, Solomon Islands: issues for successful vector control
}

\author{
Hugo Bugoro ${ }^{1 *}$, Jeffery L Hii ${ }^{2}$, Charles Butafa ${ }^{1}$, Charlie Iro'ofa', Allen Apairamo ${ }^{1}$, Robert D Cooper ${ }^{3}$,
} Cheng-Chen Chen $^{4}$ and Tanya L Russell ${ }^{5}$

\begin{abstract}
Background: The north coast of Guadalcanal has some of the most intense malaria transmission in the Solomon Islands. And, there is a push for intensified vector control in Guadalcanal, to improve the livelihood of residents and to minimize the number of cases, which are regularly exported to the rest of the country. Therefore, the bionomics of the target vector, Anopheles farauti, was profiled in 2007-08; which was after 20 years of limited surveillance during which time treated bed nets (ITNs) were distributed in the area.

Methods: In three villages on northern Guadalcanal, blood-seeking female mosquitoes were caught using hourly human landing catches by four collectors, two working indoors and two outdoors, from 18.00-06.00 for at least two nights per month from July 2007 to June 2008. The mosquitoes were counted, identified using morphological and molecular markers and dissected to determine parity.

Results: Seasonality in vector densities was similar in the three villages, with a peak at the end of the drier months (October to December) and a trough at the end of the wetter months (March to May). There was some variability in endophagy (indoor biting) and nocturnal biting (activity during sleeping hours) both spatially and temporally across the longitudinal dataset. The general biting pattern was consistent throughout all sample collections, with the majority of biting occurring outdoors (64\%) and outside of sleeping hours (65\%). Peak biting was 19.00-20.00. The proportion parous across each village ranged between 0.54-0.58. Parity showed little seasonal trend despite fluctuations in vector densities over the year.

Conclusion: The early, outdoor biting behaviour of An. farauti documented 20 years previously on north Guadalcanal was still exhibited. It is possible that bed net use may have maintained this biting profile though this could not be determined unequivocally. The longevity of these populations has not changed despite long-term ITN use. This early, outdoor biting behaviour led to the failure of the eradication programme and is likely responsible for the continued transmission in Guadalcanal following the introduction of ITNs. Other vector control strategies which do not rely on the vector entering houses are needed if elimination or intensified control is to be achieved.
\end{abstract}

Keywords: Solomon Islands, Parity, Endophagy, Biting profile, Anopheles farauti

\footnotetext{
* Correspondence: bugorohugo@yahoo.com

${ }^{1}$ National Vector Borne Disease Control Programme, Ministry of Health,

Honiara, Solomon Islands

Full list of author information is available at the end of the article
} 


\section{Background}

Malaria remains a major public health issue in the Solomon Islands [1,2]. Attempts at eradication in the 1960 s and 1970s greatly reduced malaria incidence but the programme was abandoned when it was realized that countrywide eradication was not obtainable after the main vector, Anopheles farauti, developed behavioural resistance $[3,4]$. With the collapse of vector control, transmission rates resurged until insecticide treated nets (ITN) were introduced in 1992-1993 [5]. This intervention measure resulted in a reduction in malaria cases from a high of 450 cases per 1000 people in 1992 to 150 cases per 1000 people in 1999 [6]. More recently, in 2008, the Solomon Islands government refocused the National Vector Borne Disease Control Programme (NVBDCP) to implement intensified countrywide control and regional elimination with financial backing from the Global Fund and AusAID. The key vector control tools are again insecticide treated nets (long-lasting insecticidal nets) and indoor residual spraying (IRS) with pyrethroids rather than DDT. This rejuvenated programme further reduced the countrywide incidence of malaria to 48 cases per 1,000 population in 2011 [1]. However, there exist large variations in malaria incidence between and within provinces $[2,6,7]$. One of the most malarious areas in the country is Guadalcanal Province [1] which has historically been a problem area [8] and where in 2011 there were 87 cases per 1000 people (Ministry of Health, unpublished data).

Understanding the behaviour of the local vectors is essential for planning vector control activities. The primary vector control tools, ITN and IRS, depend on mosquitoes biting indoors, late in the night and resting indoors after feeding. There are three species of malaria vectors in the Solomon Islands: Anopheles punctulatus, Anopheles koliensis and Anopheles farauti [9]. The malaria eradication programme of the $1960-70$ s had reduced the distribution and abundance of An. koliensis and $A n$. punctulatus, both of which were late night and highly endophagic biters [4], leaving An. farauti as the primary vector of malaria. The bionomics of $A n$. farauti in Guadalcanal Province was profiled in the early 1990s, prior to the introduction of ITNs. At this time, An. farauti occurred in large numbers, with peak biting outdoors and early in the night (21.00) and the entomological inoculation rates (EIR) was up to 1,022 infective bites/person/year [10-12]. Recent work in the elimination provinces of the Solomon Islands indicates that this early night, outdoor feeding pattern is still maintained $[13,14]$. Such early night outdoor feeding behaviour of An. farauti could potentially compromise the efficacy of the vector control programme.

The NVBDCP is driven to reduce malaria transmission in Guadalcanal to improve the livelihood of the residents, but also because large numbers of cases are continually exported to the elimination provinces. The area around Red Beach and Koli Point, about $20 \mathrm{~km}$ east of Honiara, was used extensively in the late 1980 s to early 1990 s to study the bionomics of An. farauti and to trial the comparative effectiveness of DDT-IRS and pyrethroid ITN $[5,10-12,15]$. More recent faunal surveys have verified that An. farauti remains very common along the north coast of Guadalcanal [16,17]; however for 20 years no studies profiled the bionomics of the vector. During this time frame ITNs were introduced into the area (in 1992-1993) and distribution and re-treatment activities were completed annually by the NVBDCP (Ministry of Health, unpublished data). The annual coverage rates varied depending on the availability of funds and political stability; nonetheless there was a continual presence of ITNs in the area. The hypothesis for this study questioned if the modified feeding behaviour of An. farauti observed after the use of DDT-IRS had been maintained over time. Such information is fundamental to conducting successful elimination or intensified control programmes.

\section{Methods \\ Study site}

The study was conducted in northern Guadalcanal in three coastal villages: Red Beach (E, 160 06.872'; S, 09 25.791'), Gilutae (E, 160 07.957'; S, 09 ${ }^{\circ} 25.206^{\prime}$ ) and Komuporo (E, $160^{\circ} 09.771^{\prime}$; S, 09²4.755') (Figure 1). The study area is $20 \mathrm{~km}$ east of the capital Honiara and encompasses numerous rural villages scattered throughout bushland on a low lying coastal plain that is cut by numerous creeks and rivers, which end on the coast in brackish swamps and lagoons. The climate of the region is continuous hot wet with an annual rainfall of 2,500 mm (median of 20 years) [18]. Rain falls throughout the year, however there is some seasonality with the months December to May receiving higher rainfall than June to November. The mean annual temperature on the coast is $26^{\circ} \mathrm{C}$ and is constant throughout the year with daily fluctuations greater than any annual fluctuations.

\section{Human landing catches (HLC)}

Seasonality, biting densities and biting behaviour were ascertained by human landing catches (HLC) conducted from 18.00-06.00 at least twice monthly from July 2007 to June 2008 in each village. Sampling was not conducted in February 2008 due to flooding. Catches were made by four collectors, two working indoors and two working outdoors, for $40 \mathrm{~min}$ each hour; all mosquitoes coming to bite the collectors' exposed feet and legs were caught using a torch and aspirator. The first team of four collectors worked from 18.00 to 00.00 and the second team of four collectors worked from 00.00 to 06.00 . Mosquitoes were held in individual waxed paper cups for each hour and location. The collectors were rotated 


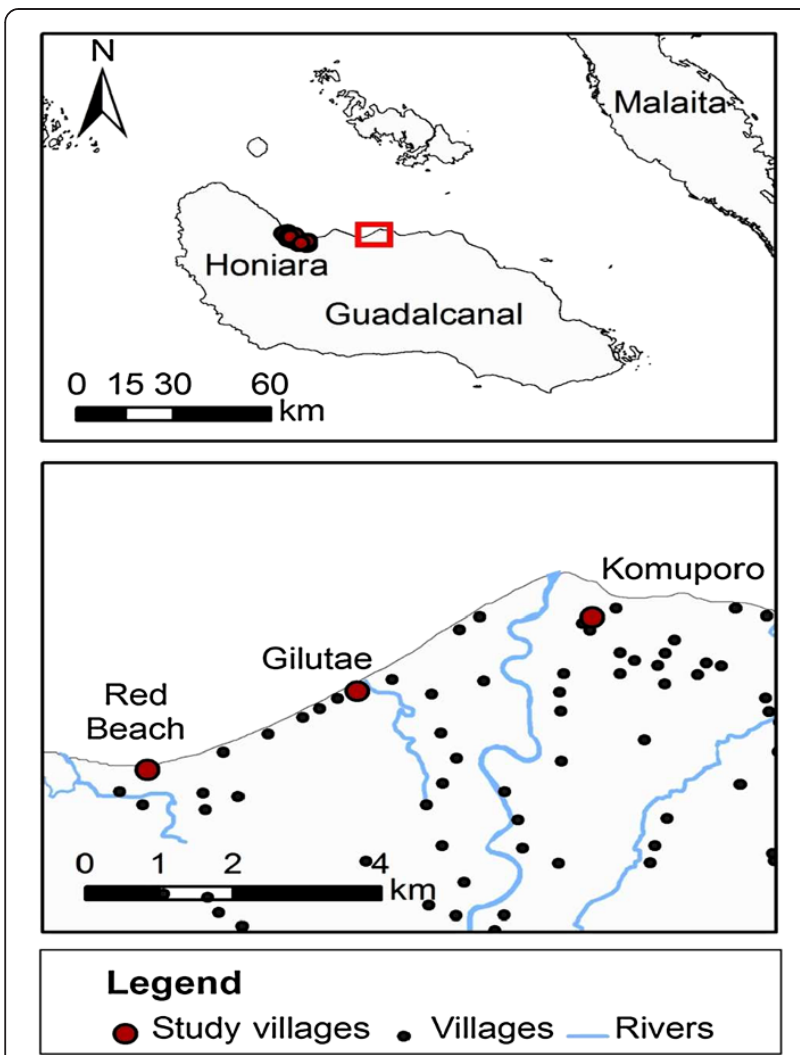

Figure 1 Map showing the location of the study villages. Top: Guadalcanal Island. Bottom: North Guadalcanal showing the three study villages and their proximity to other villages.

through the collection sites to compensate for differences in individual odours and collecting abilities. The following morning, mosquitoes were killed, counted and dissected to determine parity [19]. All Anopheles were identified by morphology [20]. Specimens were desiccated and preserved on silica gel. A subset of the specimens collected were identified by molecular analysis of the Internal Transcribed Spacer Region II of the ribosomal DNA [21] on a subset of the entire collection. In Guadalcanal, An. farauti has been shown to be the only member of the An. farauti complex which feeds on humans [9], hence the subset was analysed to confirm this.

\section{Biting behaviour}

The biting behaviour of An. farauti was compared using: 1) propensity to bite indoors (endophagy), and 2) propensity to bite during sleeping hours (nocturnal biting). The degree of endophagy was calculated as the proportion of mosquitoes biting indoors as follows:

$$
I_{18.00 \rightarrow 05.00} /\left(I_{18.00 \rightarrow 05.00}+O_{18.00 \rightarrow 05.00}\right)
$$

where $I=$ the total number of mosquitoes caught indoors, $O=$ the total number of mosquitoes caught outdoors and the subscripts represent the start time for each hour [22]. Nocturnal biting was calculated as the proportion of mosquitoes biting either indoors or outdoors during peak sleeping hours (hours starting $9 \mathrm{pm}$ to $4 \mathrm{am}$ ) as follows [22]:

$$
\left(I_{21.00 \rightarrow 04.00}+O_{21.00 \rightarrow 04.00}\right) /\left(I_{18.00 \rightarrow 05.00}+O_{18.00 \rightarrow 05.00}\right) .
$$

\section{Survival}

The ovaries of mosquitoes caught in the night landing catches were dissected in physiological saline, allowed to dry and examined under 100-200X for the presence or absence of skeins at the end of the trachea [19]. From this the proportion parous $(\mathrm{P})$ was used to determined the survival through one day $(p)$ as ${ }^{x} \sqrt{\mathrm{P}}$; where $x$ is the length of the gonotrophic cycle [23].

\section{Statistics}

The dataset was constructed with two tables: 1) field collections, and 2) parity dissections [24]. Statistical differences in mosquito biting rates between the study villages were compared using a generalized linear mixed model (GLMM) with a negative binomial distribution and a random factor to account for sample period. The differences in endophagy, nocturnal biting and parity were determined using a binomial generalized linear model (GLM) with an explanatory variable for study period, village or collection time. All analyses were conducted using the $R$ package V2.14.2.

\section{Ethics}

For this research, ethical approval was granted from the Solomon Islands Ministry of Health for conducting human landing catch as a routine programmatic activity.

\section{Results}

\section{Species identification}

All mosquitoes collected by HLC $(n=3,405)$ were determined to be An. farauti s.l. by morphology. A subset of them were confirmed to be An. farauti s.s. by molecular analysis ( $n=543 \mathrm{PCR}$ amplifications).

\section{Seasonality and vector densities}

The average biting densities of An. farauti over the year differed between the three villages $(\beta=0.165$, se $=0.077$, $p=0.036)$. The biting rate at Red Beach (17.07 bites/ person/night $(\mathrm{b} / \mathrm{p} / \mathrm{n}))$ was higher than at Komporo $(10.80 \mathrm{~b} / \mathrm{p} / \mathrm{n})$ and Gilutae $(12.09 \mathrm{~b} / \mathrm{p} / \mathrm{n})$ (Table 1). These differences in biting rates reflect the variation in productivity larval sites that were available around each village [17]. The seasonal trend was the same for each 
Table 1 The entomological estimation of the feeding behaviour and survival rates of Anopheles farauti from three villages on Northern Guadalcanal, Solomon Islands during July 2007 to June 2008

\begin{tabular}{|c|c|c|c|c|}
\hline Entomological parameters & Gilutae & Komporo & Red Beach & Overall \\
\hline \multicolumn{5}{|l|}{ Biting rate $(\mathrm{B}: \mathrm{b} / \mathrm{p} / \mathrm{n})$} \\
\hline Indoor & 7.00 & 6.62 & 15.8 & 9.66 \\
\hline Outdoor & 17.18 & 14.98 & 19.09 & 17.08 \\
\hline Overall & 12.09 & 10.80 & 17.07 & 13.40 \\
\hline Endophagy ${ }^{1}$ (Proportion indoors \pm se) & $0.29 \pm 0.014(n=1,064)$ & $0.31 \pm 0.015(n=907)$ & $0.44 \pm 0.013(n=1,434)$ & $0.36 \pm 0.008(n=3,405)$ \\
\hline Nocturnal biting ${ }^{2}$ (Proportion 21.00-05.00 \pm se) & $0.31 \pm 0.014(n=1,064)$ & $0.35 \pm 0.016(n=907)$ & $0.39 \pm 0.013(n=1,434)$ & $0.35 \pm 0.008(n=3,405)$ \\
\hline Parity (Proportion parous) (n/total) & $0.542(552 / 1,017)$ & $0.577(523 / 906)$ & $0.541(764 / 1,412)$ & $0.551(1,839 / 3,335)$ \\
\hline
\end{tabular}

$\mathrm{B}=$ no. of mosquitoes collected/no. of nights/no. of collectors.

${ }^{1}$ Proportion of mosquitoes caught indoors calculated as: $I_{18.00 \rightarrow 06.00} /\left(I_{18.00 \rightarrow 06.00}+O_{18.00 \rightarrow 06.00}\right)$; where $I=$ the total number of mosquitoes caught indoors, $O=$ the total number of mosquitoes caught outdoors and the subscripts represent the time for each hour.

${ }^{2}$ Proportion of mosquitoes caught during hours when most people are asleep calculated as: $\left(I_{22.00} \rightarrow 05.00+O_{22.00} \rightarrow 05.00\right) /\left(I_{18.00} \rightarrow 06.00+O_{18.00} \rightarrow 06.00\right)$.

village with vector densities peaking at the end of the drier months of October $(56.9 \mathrm{~b} / \mathrm{p} / \mathrm{n})$, November $(32.5 \mathrm{~b} / \mathrm{p} / \mathrm{n})$ and December $(51.3 \mathrm{~b} / \mathrm{p} / \mathrm{n})$ with the mean biting density over this three month period being $46.9 \mathrm{~b} / \mathrm{p} / \mathrm{n}$. Vector densities fell in February to their lowest at the end of the wettest months: March $(26.4 \mathrm{~b} / \mathrm{p} / \mathrm{n})$, April $(9.6 \mathrm{~b} / \mathrm{p} / \mathrm{n})$ and May $(15.8 \mathrm{~b} / \mathrm{p} / \mathrm{n})$, with a mean biting density for this three month period of $17.3 \mathrm{~b} / \mathrm{p} / \mathrm{n}$ (Figure 2).

\section{Biting behaviour}

The degree of endophagy (indoor biting) varied over the sample periods $(\beta=-0.057$, se $=0.011, p<0.001)$ and

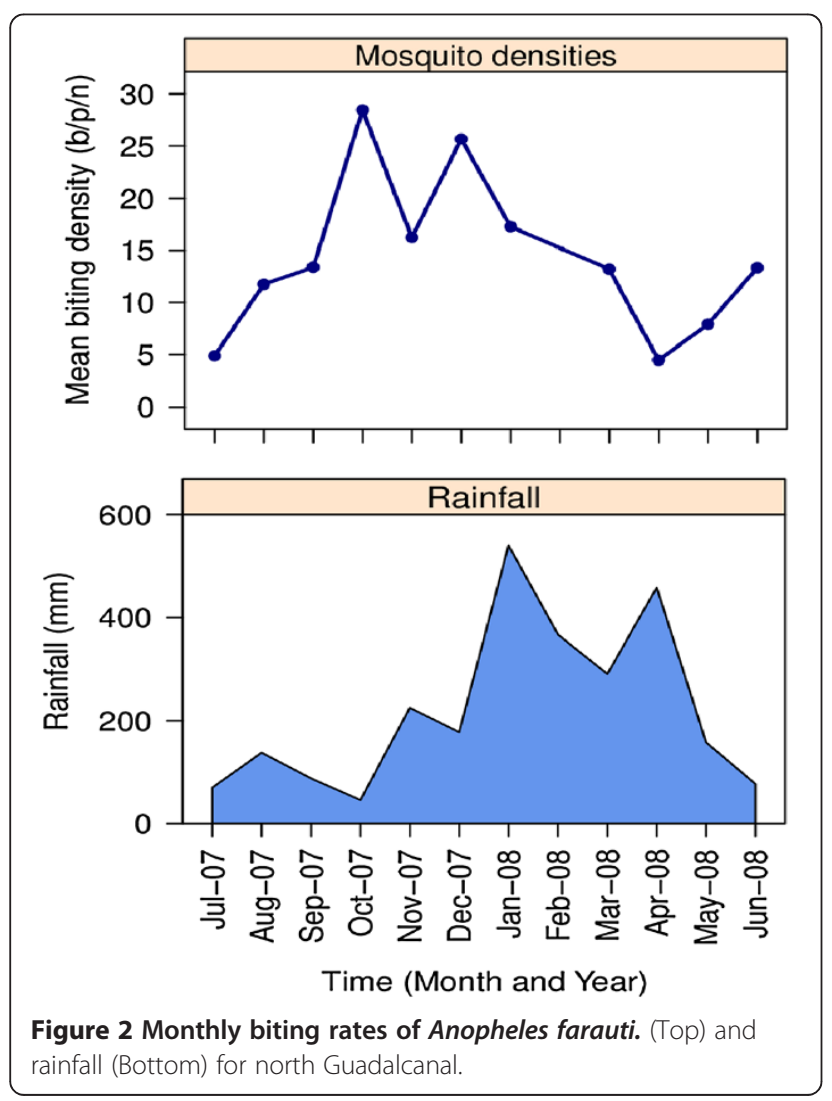

also between villages $(\beta=0.372$, se $=0.043, p<0.001)$. Endophagy was $0.29 \pm 0.014$ for Gilutae, $0.31 \pm 0.015$ for Komporo and $0.44 \pm 0.013$ for Red Beach (Table 1). Similarly the degree of nocturnal biting (activity during sleeping hours) varied over the sample periods $(\beta=0.022$, $\mathrm{se}=0.011, p=0.048)$ and also between villages $(\beta=0.187$, se $=0.042, p<0.001)$. Nocturnal biting was $0.31 \pm 0.014$ for Gilutae, $0.35 \pm 0.016$ for Komporo and $0.39 \pm 0.013$ for Red Beach (Table 1). Such levels of variation in the biting profiles are reasonable in field populations, and the general biting pattern was consistent throughout all sample collections. Overall, the majority of biting occurred outside of houses $(64 \%)$ and outside of sleeping hours (65\%) when people are unprotected by LLINs and/or IRS. Biting commenced early in the evening (at dusk 18.30) and rose rapidly to a peak in the second hour of the night (19.00$20.00)$ it then declined throughout the rest of the night to a low at 02.00-03.00 (Table 2, Figure 3). A minor increase in biting activity occurred during the two hours before dawn (04.00-06.00) (Figure 3). More than half (59\%) of all host seeking occurred during the first three hours of the night (18.00-21.00).

\section{Survival}

Dissections to measure parity were made on over 100 mosquitoes each month except for July 2007 where only 39 were dissected; in all 3,335 An. farauti were dissected over the year. The proportion parous did not vary between villages $(\beta=0.019$, se $=0.041, p=0.632)$ and showed no seasonality over the year $(\beta=-0.001$, se $=0.011, p=0.903)$. Overall the mean parity rate was $55.1 \%(1,839 / 3,335)$. With a gonotrophic cycle of 2.3 days [10], the probability of survival through one day was $79 \%$.

The only heterogeneity noted was a variation in parity throughout the night $(\beta=-0.032$, se $=0.015, p=0.032)$, with the first hour of the night having a lower proportion parous $(43.3 \%)$ than the rest of the night. However, it is unlikely that this phenomenon would have any epidemiological relevance considering the early-biting cycle 
Table 2 The parity and biting profile of Anopheles farauti compared for each hour of the night

\begin{tabular}{|c|c|c|c|c|c|c|}
\hline \multirow[b]{2}{*}{ Time } & \multirow[b]{2}{*}{ Parity } & \multirow[b]{2}{*}{$\mathrm{n} /$ total } & \multicolumn{2}{|c|}{$\begin{array}{l}\text { Mean biting } \\
\text { rate }(b / p / h)\end{array}$} & \multicolumn{2}{|c|}{$\begin{array}{l}\text { Mean parous } \\
\text { biting rate } \\
(\mathrm{b} / \mathrm{p} / \mathrm{h})\end{array}$} \\
\hline & & & Indoor & Outdoor & Indoor & Outdoor \\
\hline $18.00-19.00$ & 0.433 & $213 / 492$ & 0.984 & 2.823 & 0.426 & 1.222 \\
\hline $19.00-20.00$ & 0.517 & 478/925 & 2.302 & 5.125 & 1.189 & 2.648 \\
\hline $20.00-21.00$ & 0.574 & $332 / 561$ & 1.587 & 2.875 & 0.911 & 1.650 \\
\hline $21.00-22.00$ & 0.567 & $166 / 293$ & 0.746 & 1.586 & 0.423 & 0.899 \\
\hline $22.00-23.00$ & 0.581 & $129 / 222$ & 0.683 & 1.078 & 0.397 & 0.626 \\
\hline $23.00-00.00$ & 0.663 & $114 / 172$ & 0.548 & 0.828 & 0.363 & 0.549 \\
\hline 00.00-01.00 & 0.678 & $99 / 146$ & 0.548 & 0.594 & 0.371 & 0.403 \\
\hline $01.00-02.00$ & 0.670 & $61 / 91$ & 0.357 & 0.375 & 0.239 & 0.251 \\
\hline 02.00-03.00 & 0.623 & $43 / 69$ & 0.310 & 0.242 & 0.193 & 0.151 \\
\hline 03.00-04.00 & 0.767 & $56 / 72$ & 0.381 & 0.211 & 0.292 & 0.162 \\
\hline 04.00-05.00 & 0.550 & $60 / 109$ & 0.444 & 0.523 & 0.245 & 0.288 \\
\hline 05.00-06.00 & 0.538 & $98 / 182$ & 0.794 & 0.781 & 0.427 & 0.421 \\
\hline Total & 0.551 & 1,839/3,335 & 0.807 & 1.422 & 0.412 & 0.727 \\
\hline
\end{tabular}

of An. farauti. The mean biting rate of parous mosquitoes throughout the night was calculated by adjusting the total biting rate by the proportion parous. It is evident that the majority of human exposure to parous mosquitoes occurs before 21.00.

\section{Discussion}

This study observed a distinct seasonality in adult $A n$. farauti densities. It is important that this seasonality is considered when planning the timing of vector control activities by the NVBDCP; in particular it would be advantageous if annual activities were completed before the peak in biting occurs towards the end of the year. This seasonality reflects the larval ecology of this species

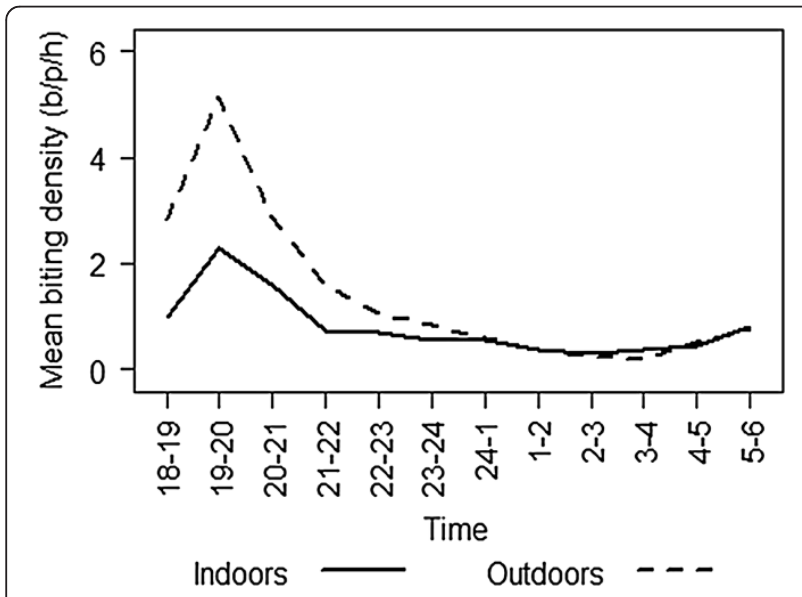

Figure 3 The hourly indoor and outdoor biting profile of Anopheles farauti from 18.00 to 06.00 in north Guadalcanal. and its ability to utilize brackish water lagoons for oviposition $[25,26]$. A study of the larval ecology in the study villages was simultaneously conducted [17], which demonstrated that larval presence and density also varied seasonally and was primarily driven by rainfall. Larval abundance was highest in the drier months when brackish lagoons formed at the mouth of the streams behind sandbars. In this supporting study [17], the peak in larval abundance occurred from September to December and would have supported the higher adult densities observed at this time. When rainfall was high (January to April), the sandbars at the mouth of the streams were washed away and in the following month the density of both larvae and adults was lower. The negative association of severe rainfall with reduced larval and adult densities of $A n$. farauti is supported by previous studies from both Vanuatu [27] and Papua New Guinea [28].

In the current study, the populations of An. farauti in Guadalcanal were observed to feed primarily outdoors and early in the evening. During the original eradication programmes of the late 1960s and early 1970s, it was observed that An. farauti avoided exposure to DDT-IRS by changing their feeding behaviour [29]. In MakiraUlawa Province prior to DDT-IRS use, the percentage of An. farauti biting before 21.00 was around $40 \%$ and there was equal feeding indoors and outdoors [29]. After DDT-IRS was implemented, the percentage of biting before 21.00 rapidly increased to more than $70 \%$ and the majority (66\%) of biting shifted to outdoors [29]. After DDT pressure was eventually removed from the mosquito populations, the modified behaviour of $A n$. farauti persisted. More recently, over the past five years, this early, outdoor biting has been observed to be sustained across the country in both Temotu [13] and Isabel Provinces [14].

Previously on northern Guadalcanal, the biting behaviour of An. farauti was profiled in 1988 [10]. The previous study was conducted after the DDT-IRS of the original eradication programme had ceased, and also before ITNs were introduced in 1993. In 1988, the peak biting time for An. farauti was 21.00-22.00 and endophagy was 30\% (range 16 to $32 \%$ ) [10]. In the current study, conducted 20 years later, the peak biting time was earlier at 19.0020.00 and endophagy was similar with $36 \%$ feeding indoors. As this change to early outdoor biting was already in place prior to the introduction of ITN it is not possible to state unequivocal that this behaviour has been maintained in An. farauti populations by the introduction of ITNs. However there is evidence that early night feeding was increased in An. farauti after only 3 weeks of ITN use in an area which had previously had none or very little DDT-IRS [30]. Also following the implementation of elimination efforts using ITNs and pyrethroids- IRS in Temotu 
Province, a lower portion of mosquitoes sought meals after 21.00 [13]. This persistence in early, outdoor biting will allow malaria to be maintained as is evident in the cases of malaria reported over the last 20 years [1]. As such there is a need to develop an integrated vector control programme which utilizes complementary strategies that consider the subtleties of mosquito ecology to further reduce the density of the local vector populations and associated malaria transmission. The most promising complementary tool which is currently available is larval control; other complementary tools generally remain at the proof of principle stage and there is a need to prioritize research funding to facilitate investigations of potential efficacy before they can be adapted into programmatic use.

Interestingly, there are indications that the behaviour of $A n$. farauti inside of sprayed houses has also changed [5]. In the same area of northern Guadalcanal, the blood-feeding success and survival of $A n$. farauti was assessed in 1989-1991. Of those females which entered houses, collections from exit window traps showed that about half were able to successfully obtain a blood meal in either houses sprayed with DDT (52\% fed) or houses with an ITN (43\% fed) [5]. However, the 24-hour mortality rates in these mosquitoes differed significantly, with $98.2 \%$ $(\mathrm{n}=219)$ mortality of females collected from houses with ITN, but only $10.1 \%(n=24)$ mortality of females collected from DDT-sprayed houses. This indicated that $A n$. farauti was able to avoid the insecticides on the walls and leave immediately after obtaining the blood meal. It should be noted that as this time, An. farauti was susceptible to DDT and mortality when exposed in WHO susceptibility tests was 77\% [5]. Additionally, the susceptibility of An. farauti to both DDT and pyrethroids was also assessed in 2006 and 100\% mortality of wild-caught adults was recorded (Ministry of Health, unpublished data). Whether An. farauti will show the same behavioural response to IRS with pyrethroids is unknown.

The annual mean parous rate recorded in this study $(54.8 \%)$ is similar to that recorded 20 years earlier in the area both in unsprayed (55.5\%) and DDT-sprayed houses (53.6\%) [5,10]. However, in 1989-1991 the parous rates in houses provided with ITN were lower (49.9\%) than the sprayed and unsprayed houses [5], indicating that ITNs had an initial impact on the longevity of the $A n$. farauti populations; but this was not sustained, possibly due to deterioration in net quality and insecticidal efficacy. Interestingly, this survival rate from 2007-08, recorded just prior to the introduction of LLINs, appears to be higher than that recorded in other populations of $A n$. farauti in the Solomon Islands from 2008 onwards (42\% in Temotu [13] and $41 \%$ in Isabel [14]). In the current study, the parous rate was relatively stable throughout the year and did not fluctuate with the changes in $A n$. farauti densities. However, parity rate for the first hour of biting (18.00-19.00) was dominated by more nulliparous mosquitoes than the remainder of the night. This has similarly been seen in populations of An. farauti in Temotu Province [13] and Central Province (Russell et al. unpublished data) and An. punctulatus in Papua New Guinea [31]. However, for An. farauti it is unlikely that this phenomenon would have any epidemiological relevance. Considering the early-biting cycle, the majority of human exposure to parous mosquitoes - which are older and have taken multiple blood meals - still occurs before 21.00.

\section{Conclusion}

The current study describes the bionomics of the primary malaria vector in the Solomon Islands, An. farauti. Recently, the NVBDCP of the Solomon Islands refocused to implement intensified countrywide control and regional elimination. The key to a successful programme will be understanding, and responding to, the behaviours of the target vector. It was observed that $A n$. farauti has a distinct seasonal profile, with peak activity from October to December, indicating that annual vector control activities should be completed before this period. Importantly, it was observed that the early outdoor biting habit of $A n$. farauti, first observed in the study area in 1988 still persists 20 years later. With this feeding behaviour, the target mosquitoes are able to minimize exposure to ITNs and IRS. Therefore, there is a need to implement complementary tools that provide personal protection or target other bionomics' vulnerabilities that may exist outside of houses, such as in the larval stages, during mating, sugar feeding or any other aspect of the life cycle. This will not only improve the success of vector control in Guadalcanal, but will reduce the number of cases that are exported to the control provinces.

\section{Availability of supporting data}

The datasets supporting the results of this article are available in the VecNet repository: https://dl.vecnet.org/ files/db78tc04f; doi:10.7274/R0J1012M.

\section{Abbreviations}

GLM: Generalized linear model; GLMM: Generalized linear mixed model; HLC: Human landing catch; IRS: Indoor residual spray; ITN: Insecticide-treated net; LLIN: Long-lasting insecticidal net; NVBDCP: National vector Borne Disease Control Programme.

\section{Competing interests}

The authors declare that they have no competing interests.

\section{Authors' contributions}

$\mathrm{HB}, J \mathrm{LH}$, and CCC designed the study; $\mathrm{HB}, \mathrm{CB}, \mathrm{Cl}, \mathrm{AA}$, and CCC performed the fieldwork; HB, RDC and TLR analysed the data and wrote the manuscript. All authors read and approved the final manuscript. 


\section{Acknowledgements}

We thank the Director, Mr Albino Bobogare and staff of the NVBDCP Ministry of Health and Medical Services for the support in performing field work in north Guadalcanal. We thank Dr. I-Yu Tsao for technical assistance in the laboratory at the National Yang-Ming University in Taiwan, and the World Health Organization Office in Solomon Islands for the logistic support. HB was supported by grant from the Taiwan International Cooperation Development Fund and CCC was supported by a grant from the Ministry of Education, Taiwan, "Aiming for the Top University Plan" for performing field work in North Guadalcanal. This work was funded by Global Fund grant MWP-507-G05-M in 2007.

\section{Author details}

'National Vector Borne Disease Control Programme, Ministry of Health, Honiara, Solomon Islands. ' ${ }^{2}$ Malaria, Other Vector-Borne and Parasitic Diseases, Regional Office for the Western Pacific, World Health Organization, San Lazaro Hospital Compound, Manila, Philippines. ${ }^{3}$ Australian Army Malaria Institute, Gallipoli Barracks, Enoggera 4052, Australia. ${ }^{4}$ nstitute of Microbiology and Immunology, National Yang-Ming University, No 155, Sec 2, Li-Nong Street, Taipei 112, Taiwan. ${ }^{5}$ Faculty of Medicine, Health and Molecular Sciences, Queensland Tropical Health Alliance, James Cook University, Cairns 4870, Australia.

Received: 9 December 2013 Accepted: 6 February 2014 Published: 15 February 2014

\section{References}

1. World Health Organization: World malaria report 2012. Geneva: World Health Organization; 2012

2. The Ministries of Health of Vanuatu and Solomon Islands, The Pacific Malaria Initiative Survey Group, Shanks D: Malaria on isolated Melanesian islands prior to the initiation of malaria elimination activities. Malar J 2010, 9:218.

3. Avery J: A review of the malaria eradication programme in the British Solomon Islands 1970-1972. P N G Med J 1974, 17:50-60.

4. Russell TL, Beebe NW, Cooper RD, Lobo NF, Burkot TR: Successful malaria elimination strategies require interventions that target changing vector behaviours. Malar J 2013, 12:56.

5. Kere NK, Arabola A, Bakote'e B, Qalo O, Burkot TR, Weber RH, Southgate BA: Permethrin-impregnated bednets are more effective than DDT house-spraying to control malaria in Solomon Islands. Med Vet Entomol 1996, 10:145-148.

6. Over M, Bakote'e B, Velayudhan R, Wilikai P, Graves PM: Impregnated nets or DDT residual spraying? Field effectiveness of malaria prevention techniques in Solomon Islands, 1993-1999. Am J Trop Med Hyg 2004, 71(Suppl 2)214-223.

7. Harris I, Sharrock W, Bain L, Gray K-A, Bobogare A, Boaz L, Lilley K, Krause D, Vallely A, Johnson M-L, Gatton M, Shanks D, Cheng Q: A large proportion of asymptomatic Plasmodium infections with low and sub-microscopic parasite densities in the low transmission setting of Temotu Province, Solomon Islands: challenges for malaria diagnostics in an elimination setting. Malar J 2010, 9:254.

8. Paik $Y-H$, Avery JG: Problem areas in the malaria eradication programme in the British Solomon Islands. P N G Med J 1973, 17:61-67.

9. Beebe N, Russell T, Burkot T, Lobo N, Cooper R: The systematics and bionomics of malaria vectors in the southwest Pacific. In Anopheles mosquitoes - New insights into malaria vectors. Edited by Manguin S. New York: InTech; 2013:357-394.

10. Hii JLK: Antimalaria program. WHO assignment report. (WPIMAL/SOLMALI 001-E. Honiara, Solomon Islands: WHO Regional Office for the Western Pacific; 1988

11. Hii JLK, Kanai L, Foligela A, Kan SKP, Burkot TR, Wirtz RA: Impact of permethrin-impregnated mosquito nets compared with DDT house spraying against malaria transmission by Anopheles farauti and An. punctulatus in the Solomon Islands. Med Vet Entomol 1993, 7:333-338.

12. Hii JLK, Birley MH, Kanai L, Foligeli A, Wagner J: Comparative effects of permethrin-impregnated bednets and DDT house spraying on survival rates and oviposition interval of Anopheles farauti No. 1 (Diptera: Culicidae) in Solomon Islands. Ann Trop Med Parasitol 1995, 89:521-529.

13. Bugoro H, Cooper R, Butafa C, Iro'ofa C, Mackenzie D, Chen C-C, Russell T: Bionomics of the malaria vector Anopheles farauti in Temotu Province, Solomon Islands: issues for malaria elimination. Malar J 2011, 10:133.
14. Bugoro H, Iro'ofa C, Mackenzie D, Apairamo A, Hevalao W, Corcoran S, Bobogare A, Beebe N, Russell T, Chen C-C, Cooper R: Changes in vector species composition and current vector biology and behaviour will favour malaria elimination in Santa Isabel Province, Solomon Islands. Malar J 2011, 10:287.

15. Kere NK, Parkinson AD, Samrawickerema WA: The effect of permethrin impregnated bednets on the incidence of Plasmodium falciparum, in children of north Guadalcanal, Solomon Islands. Southeast Asian J Trop Med Public Health 1993, 24:130-137.

16. Beebe NW, Bakotee H, Ellis JT, Cooper RD: Differential ecology of Anopheles puntucaltus and three members of the Anopheles farauti complex of mosquitoes on Guadalcanal, Solomon Islands, identified by PCR-RFLP analysis. Med Vet Entomol 2000, 41:308-312.

17. Bugoro H, Hii J, Russell T, Cooper R, Chan B, Iro'ofa C, Butafa C, Apairamo A, Bobogare A, Chen C-C: Influence of environmental factors on the abundance of Anopheles farauti larvae in large brackish water streams in Northern Guadalcanal, Solomon Islands. Malar J 2011, 10:262.

18. Brookfield HC, Hart D: Rainfall in the tropical southwest Pacific. Canberra: Department of Geography, Publ G/3, The Australian National University; 1966.

19. World Health Organization: Manual on practical entomology in malaria. Part II. Methods and techniques, WHO Offset Publication No. 13. pp. 195. Geneva: WHO Division of Malaria and Other Parasitic Diseases; 1975:195.

20. Belkin JN: The mosquitoes of the South Pacific (Diptera, Culicidae). University of California Press: Berkeley and Los Angeles; 1962.

21. Beebe NW, Saul A: Discrimination of all members of the Anopheles punctulatus complex by polymerase chain reaction - restriction fragment length polymorphism analysis. Am J Trop Med Hyg 1995, 53:478-481.

22. Govella NJ, Okumu FO, Killeen GF: Insecticide-treated nets can reduce malaria transmission by mosquitoes which feed outdoors. Am J Trop Med Hyg 2010, 82:415-419.

23. Davidson G: Estimation of the survival-rate of anopheline mosquitoes in nature. Nature 1954, 174:792-793.

24. Bugoro H, Hii J, Butafa C, Iro'ofa C, Apairamo A, Cooper R, Chen C-C, Russell T: Bionomics of Anopheles farauti in Guadalcanal, Solomon Islands: Data archive. VecNet 2013. doi:10.7274/R0J1012M.

25. Sweeney AW: Larval salinity tolerances of the sibling species of Anopheles farauti. J Am Mosq Control Assoc 1987, 8:589-592.

26. Cooper RD, Waterson DGE, Frances SP, Beebe NW, Sweeney AW: Speciation and distribution of the members of the Anopheles punctulatus (Diptera: Culicidae) group in Papua New Guinea. J Med Entomol 2002, 39:16-27.

27. Daggy RH: The biology and seasonal cycle of Anopheles farauti on Espirtu Santo, New Hebrides. Ann Entomol Soc Am 1945, 38:3-13.

28. Charlwood JD, Graves PM, Alpers MP: The ecology of the Anopheles punctulatus group of mosqutioes from Papua New Guinea: a review of recent work. PN G Med J 1986, 29:19-26.

29. Taylor B: Changes in the feeding behaviour of a malaria vector, Anopheles farauti Lav., following the use of DDT as a residual spray in houses in the British Solomon Islands Protectorate. Trans R Entomol Soc London 1975, 127:227-292.

30. Charlwood JD, Graves PM: The effect of permethrin-impregnated bednets on a population of Anopheles farauti in coastal Papua New Guinea. Med Vet Entomol 1987, 1:319-327.

31. Bockarie MJ, Alexander N, Bockarie F, Ibam E, Barnish G, Alpers M: The late biting habit of parous Anopheles mosquitoes and pre-bedtime exposure of humans to infective female mosquitoes. Trans $R$ Soc Trop Med Hyg 1996, 90:23-25.

doi:10.1186/1475-2875-13-56

Cite this article as: Bugoro et al:: The bionomics of the malaria vector Anopheles farauti in Northern Guadalcanal, Solomon Islands: issues for successful vector control. Malaria Journal 2014 13:56. 